\title{
Erratum: Damage-free vibrational spectroscopy of biological materials in the electron microscope
}

Peter Rez, Toshihiro Aoki, Katia March, Dvir Gur, Ondrej L. Krivanek, Niklas Dellby, Tracy C. Lovejoy, Sharon G. Wolf \& Hagai Cohen

Nature Communications 7:10945 doi: 10.1038/ncomms10945 (2016); Published 10 Mar 2016; Updated 4 May 2016

The author Hagai Cohen is incorrectly omitted from the list of corresponding authors. The corresponding authors are Peter Rez and Hagai Cohen. The correct information for correspondence is: 'Correspondence and requests for materials should be addressed to P.R. (email: Peter.Rez@asu.edu) or to H.C. (email: Hagai.Cohen@Weizmann.ac.il).' 